\title{
Bipolar I disorder and expressed emotion of families: A cohort study in Japan
}

\author{
Shinji Shimodera $^{1 *}$, Yukiko Yonekura ${ }^{2}$, Sosei Yamaguchi ${ }^{3}$, Aoi Kawamura ${ }^{1}$, Masafumi Mizuno ${ }^{4}$, \\ Shimpei Inoue ${ }^{1}$, Toshi A. Furukawa ${ }^{5}$, Yoshio Mino ${ }^{6}$ \\ ${ }^{1}$ Department of Neuropsychiatry, Kochi Medical School, Kochi University, Kochi, Japan \\ ${ }^{2}$ Department of Social Welfare, Kansai University of Social Welfare, Hyogo, Japan \\ ${ }^{3}$ Department of Psychiatric Rehabilitation, National Institute of Mental Health, National Center of Neurology and Psychiatry, Tokyo, \\ Japan \\ ${ }^{4}$ Department of Neuropsychiatry, Toho University School of Medicine, Tokyo, Japan \\ ${ }^{5}$ Department of Cognitive-Behavioral Medicine, Kyoto University Graduate School of Medicine/School of Public Health, Kyoto, \\ Japan \\ ${ }^{6}$ Department of Psychiatry, Mino Clinic, Okayama, Japan \\ Email: shimodes@,kochi-u.ac.jp
}

Received 4 September 2012; revised 1 October 2012; accepted 9 October 2012

\begin{abstract}
The relationships between expressed emotion (EE) of the families and the course of bipolar disorder have been examined only in a limited number of cohort studies. No study has yet been reported from Asia. The subjects were 12 patients that had been diagnosed with bipolar I disorder according to DSM-IV and their 12 key family members. The families of the patients were interviewed using the Camberwell Family Interview (CFI) within 2 weeks of the admission of the patients, and their EE were evaluated. The patients were then followed up for 9 months after their discharge from the hospital. The patients were divided into a high-EE group and a low-EE group using the cut-off based on the number of critical comments (CC) and emotional overinvolvement (EOI), and the 9-month relapse risk was compared. When the subjects with 3 or more CC or an EOI score of 3 or more were regarded as the high-EE group, and the others as the low-EE group, the 9-month relapse risk was $100 \%(3 / 3)$ for the high EE group and $0 \%(0 / 9)$ for the low EE group. (Fisher's exact test $p=0.005) \mathrm{EE}$ based on the CFI appear to be correlated with relapse in bipolar I disorder in Japan.
\end{abstract}

Keywords: Expressed Emotion; Family; Bipolar Disorder

\section{INTRODUCTION}

The expressed emotion (EE) of families of patients has been studied in schizophrenia, and its effects on the

${ }^{*}$ Corresponding author. course of the disease have been confirmed in a number of world regions $[1,2]$. The relationships between families' EE and relapse of schizophrenia, social functions, and depressive symptoms have also been clarified in Japan, which has a different culture than Western societies [3-5]. Studies on the relationships of EE with mood disorders such as depression have also been reported [6-9], and a similar study has been reported from Japan [10].

Bipolar disorders are thought to have a weaker relationship with familial stress than other mental disorder including depression [11]. However, there have been a few EE studies that suggest that families' EE effects on the course of this illness $[7,8]$. This suggest that "stress vulnerability model" may be applicable in bipolar disorder. However, there has been no study from Asia on this topic.

Bipolar disorder is an important mood disorder. Epidemiologically, lifetime risk of bipolar I disorder has varied from $0.4 \%$ to $1.6 \%$, and it is a recurrent disorder. Life time risk of bipolar II disorder is $0.5 \%$ [12]. Social cost for the disorders has been considerable [13]. Therefore it is important to clarify factors that affect the course of the disorder.

The purpose of the current study is to evaluate the relationships between families' EE and the course of bipolar I disorder using a cohort study design in Japan.

\section{METHODS}

\subsection{Subjects and Procedure}

Consecutive patients with bipolar I disorder admitted to the department of neuropsychiatry, Kochi Medical School between April, 1997 and April, 2002 and their 
families were enrolled. The diagnosis was based on DSM-IV and ICD-10. All the patients and their family members received explanation of the study purpose and procedures and provided written consent to participate in the study. The protocol for this study was approved by the Japanese Association of Psychoeducation of Depression. This study conforms to the provisions of the Declaration of Helsinki (as revised in Edinburgh 2000).

The classical cohort study that examined the relationship between bipolar disorder and relapse found the relapse rate of 9/10 among those with high EE families and that of 7/13 among those with low EE families [7]. In order to detect this much difference at alpha $=0.05$ and power $=0.80$, we would need 25 patients in each group. However we were able to recruit only 12 patients during the 5-year study period, and this study focuses on these 12 patients and their families.

The Camberwell Family Interview (CFI) [1] was performed with key family members within 2 weeks of the patients' admission and the interviews were recorded on cassette tapes. EE was evaluated using the recorded tapes and their transcriptions with regard to critical comments (CCs), hostility $(\mathrm{H})$, emotional overinvolvement (EOI), warmth (W), and positive remarks (PRs) [14]. The evaluation was performed by investigators who were formally trained and authorized for EE evaluation. If two or more interviews were carried out within one family, the evaluation with the highest number of CCs or EOI score was regarded as the EE state of the family.

The patients were treated primarily with medications including lithium carbonate and supportive psychotherapy during hospitalization and the follow-up period after discharge. Psychiatric symptoms were evaluated on admission and discharge according to the Brief Psychiatric Rating Scale (BPRS) [15] and Hamilton Depression Rating Scale (HRS) [16].

A cohort study in which the subjects were followed up for 9 months after discharge of the patients was carried out. Evaluation of symptoms by the BPRS and HRDS was performed if the attending physician suspected exacerbation of symptoms or if there were changes in the therapeutic circumstances such as readmission. The disease was regarded as having relapsed when the findings in an interview were consistent with the characteristics of a major depressive or manic episode according to DSMIV. Trained psychiatrists who were blind to the results of the EE evaluation performed these symptom evaluations.

\subsection{Analysis}

The distribution of the EE of the families studied was evaluated. Also, the subjects with 3 or more CCs or an EOI score of 3 or higher were classified as the high-EE group, and the characteristics of patients were compared between the high- and low-EE groups.
The subjects were classified into 2 groups according to the number of $\mathrm{CC}$ with the cut-off points varying from 1 to 3 , and the risk of relapse during the 9 months was compared. When EOI was 3 or more, the patient was classified into the high EE group. Given the small sample size, exact tests were carried out using the SPSS 9.0 for Windows.

\section{RESULTS}

During the study period, 12 patients with ICD-10 bipolar disorder (re-diagnosed as DSM-IV bipolar I disorder according to their case notes) and their 12 family members were entered into the study. Of the 12 patients, 8 $(66.7 \%)$ were males and 4 were females $(33.3 \%)$, and their mean age (standard deviation) was 49.7 (13.3) years. All the patients were admitted to the hospital because of their depressive state.

From the evaluation of the EE in each of the patients' families, the distribution of $\mathrm{CC}$ is shown in the Table $\mathbf{1 .}$ The number of $\mathrm{CC}$ was most frequently 0 , accounting for $58.3 \%$. The number of $\mathrm{CC}$ was 3 or more in 3 cases $(25.0 \%)$. The $\mathrm{H}$ score was 0 in all cases. The EOI score was 3 or above in 1 subject $(9.4 \%)$, for whom the number of CC was 3 .

Table 2 shows the results of comparison of the patients' characteristics between the high EE (number of CCs $\geq 3$ or EOI score $\geq 3$ ) and low EE groups. There were no significant differences.

Table 1. Distribution of Critical Comments (CC).

\begin{tabular}{ccc}
\hline Number of CC & N & $\%$ \\
\hline 0 & 7 & 58.3 \\
1 & 2 & 16.7 \\
2 & 0 & 0 \\
3 & 2 & 16.7 \\
4 & 1 & 8.3 \\
Total & 12 & 100 \\
\hline
\end{tabular}

Table 2. Comparison of the high and low EE groups.

\begin{tabular}{lcc}
\hline & High EE $(\mathrm{n}=3)$ & Low EE $(\mathrm{n}=9)$ \\
\hline & $2(66.7)$ & $\mathrm{n}(\%)$ \\
Sex (male) & \multicolumn{2}{c}{ Mean \pm SD } \\
& $49.7 \pm 14.0$ & $49.8 \pm 11.2$ \\
$\begin{array}{l}\text { Age (years) } \\
\begin{array}{l}\text { Duration of illness } \\
\text { (years) }\end{array}\end{array}$ & $8.3 \pm 4.6$ & $5.0 \pm 6.7$ \\
$\begin{array}{l}\text { BPRS score at } \\
\text { admission }\end{array}$ & $21.7 \pm 9.1$ & $20.2 \pm 8.6$ \\
\hline
\end{tabular}

No significant difference by Fisher's test (2-tailed) or t-test (2-tailed); BPRS: Brief Psychiatric Rating Scale; High EE: (3 or more CC) or (3 or more EOI); CC: Critical comments; EOI: Emotional overinvolvement. 
Table 3 shows a comparison of the number of previous manic/depressive episodes according to EE. In the high EE group raw figures are presented, as the subject number was too small.

In Table 4, the association between EE and relapse is shown. When we adopted $3 \mathrm{CCs}$ as the cutoff point, the 9-month relapse risk was $100 \%(3 / 3)$ for the high EE group and $0 \%(0 / 9)$ for the low EE group (Fisher's exact test $\mathrm{p}=0.005$ ), with $100 \%$ sensitivity and specificity. Two of the 3 were defined as having relapsed into a manic state, and 1 as having relapsed into a depressive state. When we used only CCs for the analysis, the result was the same as in Table 4. A similar analysis only for EOI was impossible because of the small number of the subjects scoring 2 or 3 for EOI.

\section{DISCUSSION}

We will briefly review the previous literature. Miklowitz et al. conducted a cohort study to investigate the association between the course of bipolar disorder and EE, affective style, and lithium therapy among 24 patients [7].

Table 3. Comparison of past episodes by high and low EE.

\begin{tabular}{lccc}
\hline & Depressive & Manic & Total numbers \\
\hline \multicolumn{3}{c}{ Mean (range) } \\
\hline Low EE $(\mathbf{n}=\mathbf{9})$ & $1.7(1-3)$ & $1.9(1-6)$ & $3.6(2-8)$ \\
High EE $(\mathbf{n}=3)^{*}$ & $1(1-1)$ & $2(1-4)$ & $3(2-5)$ \\
\hline
\end{tabular}

High EE: (3 or more CC) or ( 3 or more EOI); CC: Critical comments; EOI: Emotional overinvolvement.

*in detail

\begin{tabular}{cccc}
\hline \multicolumn{4}{c}{ High EE $\left(\mathbf{n}=\mathbf{3}^{*}\right.$} \\
\hline & Depressive & Manic & Total \\
\hline Case 1 & 1 & 1 & 2 \\
Case 2 & 1 & 4 & 5 \\
Case 3 & 1 & 1 & 2 \\
\hline
\end{tabular}

Table 4. Families' EE and relapse of bipolar disorder.

\begin{tabular}{ccccc}
\hline & & \multicolumn{2}{c}{ Relapse (\%) } & \multirow{2}{*}{ Total } \\
\cline { 3 - 4 } & & None & Relapsed & \\
\hline \multirow{2}{*}{ EEE1 $^{* 1}$} & Low & $7(100)$ & $0(0)$ & 7 \\
& High & $2(40)$ & $3(60)$ & 5 \\
& Total & $9(75)$ & $3(25)$ & 12 \\
& Low & $9(100)$ & $0(0)$ & 9 \\
EEE2 $^{* 2}$ & High & $0(0)$ & $3(100)$ & 3 \\
& Total & $9(75)$ & $3(25)$ & 12 \\
\hline
\end{tabular}

${ }^{* 1} \mathrm{p}=0.045$ by Fisher's exact test, sensitivity $=100 \%$, specificity $=77.8 \%$. ${ }^{*} \mathrm{p}=0.005$ by Fisher's exact test, sensitivity $=100 \%$, specificity $=100 \%$; High EE1: (1 or more CC) or (3 or more EOI); High EE2: (3 or more CC) or (3 or more EOI); CC: Critical comments; EOI: Emotional overinvolvement.
They found a positive association between the families' EE and relapse. Priebe et al. also conducted a cohort study in 21 patients with bipolar and schizoaffective disorders treated by lithium [8]. They observed an association between EE and relapse. Kim et al. studied the effects of families' EE on the course of 125 bipolar disordered patients [17]. They followed-up the patients for 2 years, and found no relationship between EE and relapse. In these 3 studies, the families' EE was evaluated by CFI.

On the other hand, Yan et al. used FMSS (Five-Minute Speech Sample) and conducted a 1-year cohort study among bipolar I disordered patients [18]. They found that the families' EE was related to depressive relapse.

These previous studies suggest that the family environment as evaluated by EE affects the course of bipolar disorders, although one study showed negative findings. In the current study, we support these findings and submit the first report from East Asia, where different cultures and religions exist.

Concerning the CC cut-off point, the validity was best when the subjects were divided between 3 or more and 2 or less. In this case, both sensitivity and specificity were $100 \%$. However Miklowitz et al. used $6 \mathrm{CC}$ as the cutoff point [7]. This means that, in Japan, the CC cut-off point for high EE should be changed, as families' emotional expression may be less salient in this culture.

If the high EE group has experienced more previous manic/depressive episodes than the control group, this would explain the current results. However, we found no evidence for it (Table 3).

As the relapse during the follow-up period, in the high EE group, 2 out of 3 were defined as having relapsed into a manic state, and 1 into a depressive state. Because of the small number of subjects, we could not find alternative reason for the 2 types of relapse.

Finally, we will discuss the limitations of the current study. 1) Small sample size: We could not examine the EE's effect on the bipolar I disorder compared to the bipolar II disorder; 2) The subjects were inpatients. Therefore, the findings might be valid only among more severely affected patients. Because the number of outpatients is larger than that of inpatients, further studies are required in outpatients including those with bipolar II disorder. Also studies with adolescents are required since this study was conducted mainly on middle-aged patients [19].

\section{ACKNOWLEDGEMENTS}

The authors express their thanks to the patients and their families who kindly participated in this study, and to Dr. Koichiro Sudo, Tosa Hospital for his assistance and discussion. This study was partly funded by a grant-in-aid for scientific research (B) from the Japan Ministry of Edu- 
cation, Science, Sports and Culture (grant No. 17390191).

\section{REFERENCES}

[1] Leff, J. and Vaughn, C. (1985) Expressed emotion in families. Guilford Press, New York.

[2] Butzlaff, R.L. and Hooley, J.M. (1998) Expressed emotion and psychiatric relapse, a meta-analysis. Archives of General Psychiatry, 55, 547-552. doi:10.1001/archpsyc.55.6.547

[3] Tanaka, S., Mino, Y. and Inoue, S. (1995) Expressed emotion and schizophrenic course in Japan. British Journal of Psychiatry, 167, 794-798. doi:10.1192/bjp.167.6.794

[4] Mino, Y., Inoue, S., Tanaka, S. and Tsuda T. (1997) Expressed emotion among families and course of schizophrenia in Japan: A 2-year cohort study. Schizophrenia Research, 24, 333-339. doi:10.1016/S0920-9964(96)00129-6

[5] Mino, Y., et al. (1998) Expressed emotion of families and negative/depressive symptoms in schizophrenia: A cohort study in Japan. Schizophrenia Research, 34, 159-168.

[6] Hooley, J.M., Orley, J. and Teasdale, J.D. (1986) Levels of expressed emotion and relapse in depressive patients. British Journal of Psychiatry, 148, 642-647. doi:10.1192/bjp.148.6.642

[7] Miklowitz, D., et al. (1988) Family factors and the course of bipolar affective disorder. Archives of General Psychiatry, 45, 225-231. doi:10.1001/archpsyc. 1988.01800270033004

[8] Priebe, S., Wildgrube, C. and Muller-Oerlinghausen, B. (1989) Lithium prophylaxis and expressed emotion. British Journal of Psychiatry, 154, 396-399. doi:10.1192/bjp.154.3.396

[9] Okasha, A.E.L., et al. (1994) Expressed emotion, perceived, and relapse in depression: A replication in an Egyptian community. American Journal of Psychiatry, 151, 1001-1005.
[10] Mino, Y., et al. (2001) Expressed emotion of families and the course of mood disorders: A cohort study in Japan. Journal of Affective Disorders, 63, 43-49. doi:10.1016/S0165-0327(00)00185-3

[11] Fukuzawa, K., et al. (2011) Family psychoeducation for major depression: Randomized controlled trial. British Journal of Psychiatry, 198, 385-390. doi:10.1192/bjp.bp.110.078626

[12] World Health Organization (1992) The ICD-10 classification of mental and behavioural disorders, clinical description and diagnostic guidelines. WHO, Geneva.

[13] American Psychiatric Association (1994) Diagnostic and statistical manual of mental disorders. 4th Edition, APA, Washington DC.

[14] Mino, Y., et al. (1995) Training in evaluation of expressed emotion using the Japanese version of Camberwell Family Interview. Acta Psychiatrica Scandinavica, 92, 183-186. doi:10.1111/j.1600-0447.1995.tb09565.x

[15] Overall, J.E. and Gorham, D.R. (1962) The brief psychiatric rating scale. Psychological Reports, 10, 799-812. doi:10.2466/pr0.1962.10.3.799

[16] Hamilton, M. (1967) Development of a rating scale for primary depressive illness. British Journal of Social \& Clinical Psychology, 6, 278-296. doi:10.1111/j.2044-8260.1967.tb00530.x

[17] Kim, E.Y. and Miklowitz, D.J. (2004) Expressed emotion as a predictor of outcome among bipolar patients undergoing family therapy. Journal of Affective Disorders, 82, 343-352.

[18] Yan, L.J., et al. (2004) Expressed emotion versus relationship quality variables in the prediction of recurrence in bipolar patients. Journal of Affective Disorders, 83, 199-206. doi:10.1016/i.jad.2004.08.006

[19] Macneil, C., et al. (2011) Psychological needs of adolescents in the early phase of bipolar disorder: Implications for early intervention. Early Intervention in Psychiatry, 5 100-107. doi:10.1111/j.1751-7893.2011.00273.x 